Overview of Deployed EDS Technologies And Third Party Involvement with Advancedments

H. E. Martz, C. R. Crawford

January 10, 2011 
This document was prepared as an account of work sponsored by an agency of the United States government. Neither the United States government nor Lawrence Livermore National Security, LLC, nor any of their employees makes any warranty, expressed or implied, or assumes any legal liability or responsibility for the accuracy, completeness, or usefulness of any information, apparatus, product, or process disclosed, or represents that its use would not infringe privately owned rights. Reference herein to any specific commercial product, process, or service by trade name, trademark, manufacturer, or otherwise does not necessarily constitute or imply its endorsement, recommendation, or favoring by the United States government or Lawrence Livermore National Security, LLC. The views and opinions of authors expressed herein do not necessarily state or reflect those of the United States government or Lawrence Livermore National Security, LLC, and shall not be used for advertising or product endorsement purposes.

This work performed under the auspices of the U.S. Department of Energy by Lawrence Livermore National Laboratory under Contract DE-AC52-07NA27344. 


\title{
Overview of Deployed EDS Technologies And Third Party Involvement with Advancements
}

\author{
Harry E. Martz, Jr. ${ }^{1}$ and Carl Crawford ${ }^{2}$ \\ ${ }^{1}$ Lawrence Livermore National Laboratory \\ Livermore, CA \\ ${ }^{2}$ Explosives Division, Science \& Technology Directorate \\ US Department of Homeland Security \\ Washington, DC \\ Work performed on the \\ Science \& Technology Directorate of the \\ Department of Homeland Security \\ Statement of Work \\ PR RSEN-08-00066 \\ January 11, 2011 \\ IM 461531 \\ LLNL-TR-465094
}

This work performed under the auspices of the U.S. Department of Energy by Lawrence Livermore National Laboratory under Contract DE-AC52-07NA27344.
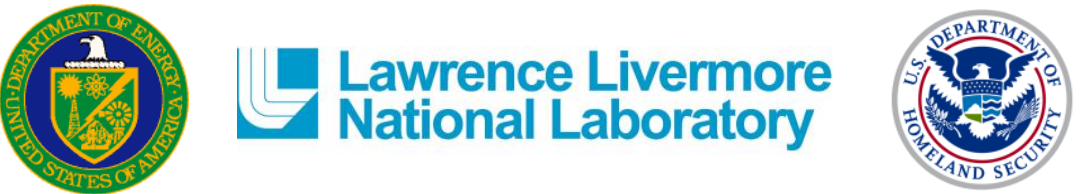


\section{Table of Contents}

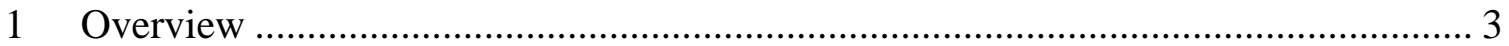

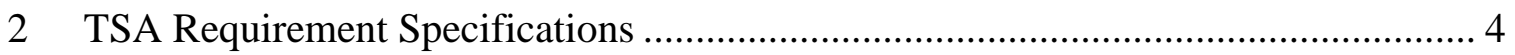

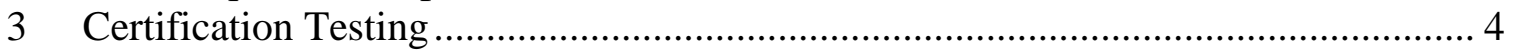

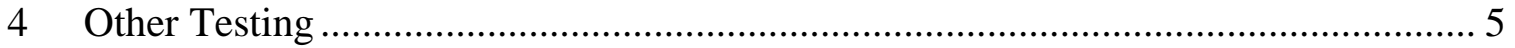

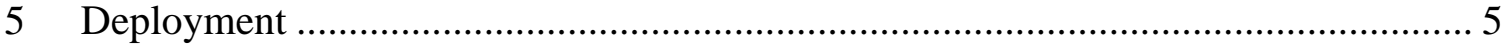

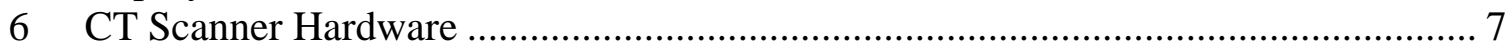

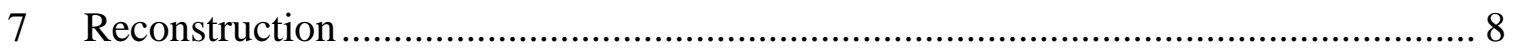

8 Dual Energy Decomposition ........................................................................... 9

9 Automated Threat Detection …………………….......................................... 10

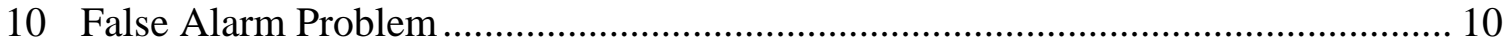

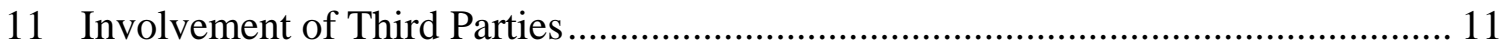

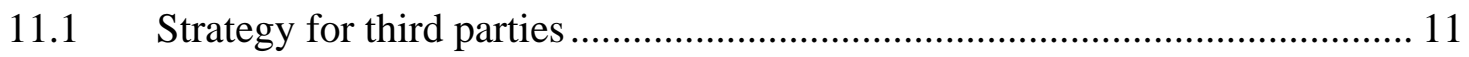

11.2 Opportunities for third parties .............................................................. 11

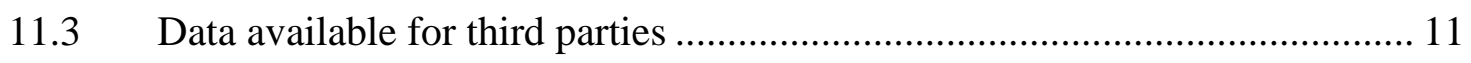

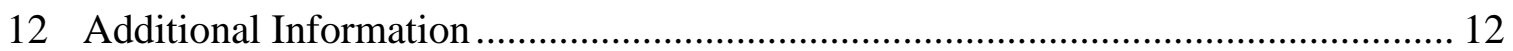

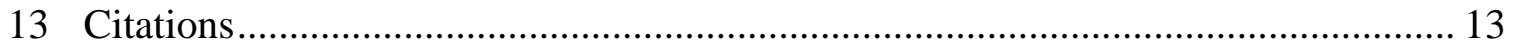

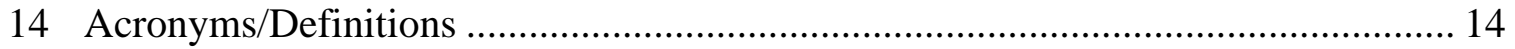

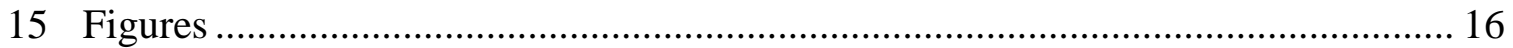




\section{Overview $^{1}$}

The term explosive detection system (EDS) is used by the TSA to describe equipment that is certified to detect explosives in checked bags. The EDS, as certified, by the TSL must consist of device for interrogating a bag and an automated detection algorithm (ATD) for evaluating the results of the interrogation. We only consider CT as the interrogation device in this report. A schematic drawing of a CT-based EDS is shown in Figure $2^{2}$.

The output of the ATD is the binary decision of alarm or non-alarm. Alarms may true- or false-positives. Non-alarms may be true- or false-negatives. False positives are also denoted false alarms. The true detection means that the ATD reports an alarm when a threat is present in the scanned bag. The probability of detecting a threat given that a threat is present is denoted the probability of detection (PD). The probability of false alarm (PFA) is the case when an alarm is reported when a threat is not present in a bag. Certification in this context means passing tests for PD and PFA at the TSL.

The results of the EDS include CT cross-sectional images of the bag and specifics about the alarmed objects generated by ATD. These results are presented on a display so that a person may override the decision of ATD and declare the alarm to be a non-alarm. This process is denoted clearing.

Bags that are not cleared by the person are sent to a secondary inspection process. Here the bags may be opened or assessed with explosive trace detection (ETD) in order to clear the bags. Bags that are not cleared at this point are evaluated by an ordinance disposal team.

The CT scanner along with ATD is denoted Level 1 screening. The process of clearing on a display is denoted Level 2 screening. Secondary inspection is denoted Level 3 screening. Vendors of the deployed EDSs supply the TSA with equipment for all three levels. Therefore, the term EDS may include the equipment provided for Levels 1, 2 and 3.

A schematic diagram of an EDS and the levels of screening are shown in Figure 7. The decision processes used as a bag is scanned and cleared is shown in Figure 8. Shield alarms and exceptions are discussed below. Since most alarms are false alarms, the probability of alarm is oftentimes denoted the probability of a false alarm. The expenses associated with clearing false alarms occur in Levels 2 and 3.

\footnotetext{
${ }^{1}$ A table with acronyms and their definitions can be found at the end of this document.

${ }^{2}$ Figures can be found at the end of this document. The figures are numbered out of order because this report was derived from multiple other documents.
} 


\section{TSA Requirement Specifications}

The TSA specifications that are relevant to the false alarm problem are discussed in this section. Some of the details are omitted because they are SSI or Classified.

An EDS must detect a number of categories of explosives. The PD for each category has to be greater than the threshold $x$. The average of the PDs for all the categories has to be greater than the threshold $y$, where $y>x$. The PFA for the EDS has to less than the threshold $z$. Detection must be performed automatically with a system denoted ATD. The humans that participate in Levels 2 and Level 3 are not tested as part of these requirement specifications.

The throughput of EDS must be at least 450 bags per hour. Multiple scanners may be configured in parallel to achieve this requirement. This throughout is measured without consideration of the TSO resolving false alarms. This condition is known as the human is not in the loop.

The EDS must report that exceptions occurred. The exceptions include cut bags, shield alarms, ATD time-outs, bag jams and scanner failures. Shield alarms are the largest source of exceptions; there is no requirement specification for shield alarms.

An EDS is also required to record data about the bags being scanned, the threats found by ATD, and the results of Level 2 and Level 3 screening. The data is collected by the FDRS.

\section{Certification Testing}

EDSs are certified to meet the above requirements at the TSL using tests that have been described in detail elsewhere [Management-Plan] $]^{3}$. A summary of the tests is as follows.

Two sets of bags were created, one with one threat per bag and one with no threats per bag. The bags are preserved so that all vendors are tested with the same sets of bags. The bags are not representative of bags in the field because fragile, perishable and valuable items are not included. These test sets are not moved from the TSL so that testing could be performed at the factories of the vendors or on fielded systems.

The bags with threats are run through the EDS and the PD per category and the average of the PDs per category are calculated by summing the binary decision reported by ATD. The bags without threats are scanned and the decision reported by ATD is summed to report PFA. Throughput is measured on the set without threats.

\footnotetext{
${ }^{3}$ The notation $[\mathrm{XXX}]$ indicates a citation to an entry in the bibliography below.
} 
The EDS that passes certification is denoted the certified system. Subsequent tests are designed to assure that all manufactured and deployed scanners match the performance of the certified system.

The following vendors have certified systems: $\mathrm{GE}^{4}$ (and its predecessor InVision), L-3 Communications, Analogic and Reveal. The majority of the 1500 systems deployed after 9/11 are produced by GE, L-3 Communication and Reveal. All of the 1500 systems are based on CT.

GE has also certified systems based on XRD. However, the throughput of these scanners is too low to be deployed. Therefore, systems based on XRD are not discussed in this section.

\section{Other Testing}

Other tests are performed at the TSL in addition to certification. CRT and pre-cert are run to qualify a system before certification. After certification, a post-certification test is run to assure that there are certain configurations and locations of explosives that are not detected. The acceptance criteria for these tests are subjective. The basis for this additional testing appears to be based on the following statement from TSA's requirements: "The detection must not be dependent on the shape, position, or orientation of the explosive or the configuration of an improvised explosive device (IED)" [Management-Plan].

EDSs are tested in a factory using a factory acceptance test (FAT) and when they are installed at a site using a site acceptance test (SAT). It is not known who controls FAT and SAT. FAT and SAT are run to assure that all systems match the certified system. However, the committee did not hear evidence to show that PD in the field matches the PD obtained during certification.

The vendors scan test phantoms (also known as test bags) periodically to assure that the scanners in the field are performing per specification. It is not known who controls the requirements for frequency of these scans. It is known that the acceptance criteria are set by the vendors.

\section{Deployment}

EDSs are deployed in a number of configurations. In-line means that the EDS given bags by the BHS. Stand-alone means that bags are fed manually. Stand-along systems can be in front or begin the check in counter.

\footnotetext{
${ }^{4}$ Since this report was initially written, GE has changed its name to Morpho Detection
} 
An in-line deployment is shown in Figure 9 and depicted in Figure 1. The blocks in this figure are now described. The process that is followed in the field is denoted SOP.

An EDS consists of the following components: (1) CT scanner; (2) automated threat detection (ATD) algorithm; (3) baggage viewing station (BVS) and (4) control computer (CC). The EDS is integrated with the following other components: (5) baggage handling system (BHS); (6) baggage inspection room (BIR) and (7) ordinance disposal team (ODT).

The CT scanner produces cross-sectional images of the bags. The images are either a set of contiguous slices, known as 3D or volumetric data, or a variable number of slices at varying slice spacing, known is selective slices. The CT scanner may be combined with an x-ray line scanner, which also denoted a digital radiography (DR) projection scanner. The images from the DR are used to determine where to acquire the selective slices.

The ATD processes the images produced by the CT scanner to locate threats. Zero or more threats may be found per bag. The output of the ATD includes descriptions of the threats including their locations within in a bag. Cleared bags (bags with no threats found by the ATD) are sent to the airplane. The performance of an ATD is characterized by its PD and PFA. PD is less than $100 \%$ and PFA is greater than $0 \%$. ATD may run on computers in the CT portion of the EDS or on the BVS.

The ATD also analyzes the images of the bags and determines if a threat could be shielded from the X-rays used in the EDS. If shielded regions are found in the bag, the bag and its images are sent directly to the BIR.

The BVS displays images of bags that contain threats. A TSO may clear the decision of ATD using a protocol known as OSR or OSARP. The procedure used by the TSO during OSR is SSI. Bags cleared by the TSO per OSR are sent to the airplane. If ATD finds multiple threats, the TSO may clear some or all of the threats. The BVS is also known as a PVS. The use of the BVS is also denoted Level 2 screening.

The BIR receives bags that have not been cleared by the TSO using OSR on the BVS. TSOs visually inspect the threats or apply explosive trace detection (ETD) to attempt to clear threats. If the TSO clears the threats, the bag is sent to the airplane. Bags with remaining threats are transferred to an ordinance disposal team (ODT). The processing in the BIR is known as Level 3 screening. There may be a workstation, denoted the SVS, in the BIR, where the TSO examines the CT slices and the outputs of ATD. It may be possible that the threat, as found by ATD, is not found by the TSO or another item is mistaken for the threat.

The BHS consists of a set of conveyor belts, diverting mechanisms and a tracking system. The conveyor belts move bags in and out of the EDS, to the BIR and to the airplane. The diverting mechanisms move the bags between the different sections of the conveyor belts. There are a number of exceptions, in addition to shield alarms, that may cause a bag and its images to be sent directly to the BIR. The exceptions include shield 
alarms, mis-tracking, operator time-out errors, bag jams in the scanner, and scanner failures.

\section{CT Scanner Hardware}

CT scanners collect projections from different angular positions. The projections are inverted using the step denoted reconstruction to produce cross-sectional images. The images provide estimates of the object's linear attenuation coefficient, which is closely related to physical density, and optionally the atomic number. The images are contiguous, resulting in a $3 \mathrm{D}$ volume, or selective $2 \mathrm{D}$ slices, known as selective-slice. ATD follows reconstruction. A picture of the inside of a CT scanner is shown in Figure 3. The components of the CT scanner are now described.

A typical CT scanner is described in this section. Variations are noted in the text and after the description. CT scanners have five key subsystems: HVPS, x-ray source, detector, gantry, data acquisition system, and reconstruction algorithm.

The HVPS produces high-voltages required to drive the $\mathrm{x}$-ray tube. The average potential of the HVPS is in the range $140-180 \mathrm{kV}$. Some systems use a DC waveform. Other systems add an AC component in order to collect high and low voltage information, as described below. The power of the HVPS is the range $500-5000 \mathrm{~W}$.

The x-ray tube produces a Bremsstrahlung spectrum of x-rays from $0 \mathrm{keV}$ to the peak potential of the HVPS.

The bag is transported through the EDS on a conveyor belt. The bag and the conveyor are not shown in Figure 3.

The detector detects x-rays that pass through a bag under inspection. The detector converts the x-ray photons to light. Photodiodes, which are mounted behind the detector, convert the light to electrical charge. There are one or more rows of detectors, where each row forms a fan-beam with x-ray tube as the vertex of the fan. The collection of detector rows forms a cone-beam.

The output of the detector is digitized by the DAS. The outputs of the DAS are either fanbeam or cone-beam projections. These projections are related to the line-integrals of the $\mathrm{x}$-ray attenuation coefficient of the bag along the paths from the $\mathrm{x}$-ray tube to the detectors. These projections are denoted raw data in the parlance of CT scanners. They are denoted raw projection data in the parlance of CT-based EDS.

The x-ray tube, HVPS, detector and the DAS are mounted on a gantry. The gantry rotates at approximately 0.5 seconds per rotation. The DAS is sampled at approximately a $1 \mathrm{kHz}$ so that projections are obtained at various angular positions around the bag. 
The conveyor belt may or may not be stationary when the gantry rotates around the bag. The scanner is considered to be a step-and-shoot variety if the conveyor is stationary. The scanner is considered to be a helical or spiral scanner if the conveyor is moving.

The outputs of the DAS are sent to a reconstruction computer to reconstruct crosssectional images. The cross-sectional images are sent to another computer on which ATD is performed. The reconstruction and ATD computers are not shown in Figure 3.

Some EDSs combine CT with a DR scanner. The images from the DR are used to determine where selective cross-sectional images should be acquired. The deployed GE scanners use this combination.

The GE (InVision) scanners combine DR with a step-and-shoot CT scanner. Reveal and L-3 only use volumetric CT scanners. GE (InVision) and L-3 are single energy systems and therefore only produce measurements of the linear attenuation coefficient. The Reveal system obtains dual energy measurements and therefore can produce estimates of the atomic number.

\section{Reconstruction}

Most scanners use a process denoted filtered-back-projection (FBP) to reconstruct the cross-sectional images [Kak-Slaney]. The output of the DAS is corrected in order to generate the line-integral data required by FBP. The steps of reconstruction are shown schematically in Figure 4.

If the steps in correction cannot completely reverse the underlying physical effects, images will be degraded leading to inaccurate measurements of the linear attenuation coefficient, density and atomic number. The following operations may be performed during the correction step.

\begin{tabular}{|l|l|}
\hline \multicolumn{1}{|c|}{ Step } & \multicolumn{1}{c|}{ Synopsis } \\
\hline Offset & $\begin{array}{l}\text { The electronics (photodiode and amplifiers in the DAS) have dark currents. } \\
\text { The dark currents are measured with the x-ray tube off and then subtracted. } \\
\text { Temperature drift of the offset has to be considered. }\end{array}$ \\
\hline Reference & $\begin{array}{l}\text { The current supplied by the HVPS to the x-ray tube may vary. A reference } \\
\text { detector measures the incident x-ray flux. }\end{array}$ \\
\hline Beam hardening & $\begin{array}{l}\text { The x-ray tube produces a polychromatic spectrum. The x-ray attenuation } \\
\text { coefficient is a function of the photon energy, with lower energy photons } \\
\text { being preferentially removed. A polynomial correction is applied. } \\
\text { Unfortunately the different materials use different polynomials so artifacts } \\
\text { will remain. }\end{array}$ \\
\hline Spectral & $\begin{array}{l}\text { Each detector has its own spectral response to polychromatic x-rays. This } \\
\text { response is known as the detector's transfer function. The difference of the } \\
\text { transfer function for each detector with respect the mean of the functions for } \\
\text { all the detectors is corrected in order to prevent the insertion of concentric } \\
\text { rings an bands in images. }\end{array}$ \\
\hline Afterglow & $\begin{array}{l}\text { The detector and DAS have finite impulse responses leading to a temporal } \\
\text { blur of the projections. The impulse responses may be de-convolved. }\end{array}$ \\
\hline
\end{tabular}




\begin{tabular}{|l|l|}
\hline Scatter & $\begin{array}{l}\text { Scattered x-ray photons may reach the detector. Some scattered photons may } \\
\text { be eliminated with anti-scatter plates placed in the septa between detectors. } \\
\text { Additional algorithmic correction can be used to remove scatter based on } \\
\text { measurements from auxiliary detectors or using the projections themselves. }\end{array}$ \\
\hline Clamping & $\begin{array}{l}\text { The DAS has a finite dynamic range, which is determined in part by the } \\
\text { electronic noise in the DAS. The number of x-ray photons reaching the } \\
\text { detector may be on the level of the electronic noise. The number of photons is } \\
\text { clamped to a positive number. However, artifacts will still be generated in } \\
\text { images when this condition occurs. }\end{array}$ \\
\hline Gain & $\begin{array}{l}\text { Each detector has its own gain. The gain is measured by scanning only air. } \\
\text { The values of the air readings are used to scale the readings through a bag. } \\
\text { The gains may be a function of the angular position of the gantry, }\end{array}$ \\
\hline Logarithm & $\begin{array}{l}\text { The DAS/detector combination integrates energy. In order to generate the line } \\
\text { integrals required by FBP, the natural logarithm of the readings has to be } \\
\text { taken. }\end{array}$ \\
\hline Re-binning & $\begin{array}{l}\text { The cone-beam projections are processed to form fan-beam or parallel-beam } \\
\text { projections. If the projections were acquired using helical scanning, then the } \\
\text { movement of the bag during data acquisition is removed using interpolation. }\end{array}$ \\
\hline
\end{tabular}

The projections that result from the correction step are denoted corrected data in the parlance of CT scanners. They are denoted corrected projection data in the parlance of CT-based EDS.

Reconstruction may also be performed using direct Fourier methods or iterative methods [Kak-Slaney]. The iterative methods are not generally used because of their computational expense relative to FBP.

\section{Dual Energy Decomposition}

The x-ray attenuation at the energies used for explosive detection is mainly determined by the Photoelectric effect and Compton Scatter. If two different readings are taken for each path from the $\mathrm{x}$-ray source to the detector, each reading with a different $\mathrm{x}$-ray spectral, then line integrals of the Photoelectric and Compton contributions can be found solving two nonlinear equations. These integrals can be reconstructed using FBP to produce images of the Photoelectric and Compton contributions. It is then possible to solve for the physical density and the effective atomic number on a voxel-by-voxel basis.

Different X-ray spectra may be generated by modulating the HVPS with an AC component or two sets of detectors can be stacked on top of each other so that one detector attenuates the photons seen by the second detector. The stacked configuration is known as sandwich detectors. The EDSs produced by Reveal are the only deployed scanners that use dual energy and they use sandwich detectors.

A detailed review of the use of dual energy for explosive detection can be found elsewhere [Ying].

Dual energy may be useful to separate threats from non-threats when only density is used by ATD. 


\section{Automated Threat Detection}

A schematic diagram of a notional ATD is shown in Figure 5. The purpose of ATD is to segment (find) objects in the cross-sectional images and then to classify if the object is a threat or a non-threat. Additional steps in ATD include compensation for imperfect correction in the CT reconstruction step and extraction of features such as density, atomic number and mass.

The density and atomic number of objects are compared to the values of known explosives. If these values are in the desired range and the mass sufficient, then the object is declared a threat. Vendors may use other features in their classification step, but these features are proprietary. CT is not specific to the chemical and molecular composition of explosives. False alarms occur when non-threats are share the same density and atomic number of threats.

Some vendors may also use the projection data and the images from the DR in their ATD algorithm. ATD may use different methods, denoted paths, for finding sheet and bulk explosives.

\section{False Alarm Problem}

False alarms will be generated if non-threats have the same density and atomic number as threats. This is shown in Figure 6 for density. The use of atomic number may be used to reduce the overlap in $2 \mathrm{D}$ space.

The correction step in CT reconstruction attempts to correct for imperfections in the projection data acquired during scanning. However, if the corrections are not perfect, then artifacts will be generated in images leading to imprecise measurements of object characteristics. This will require a broadening of the acceptance criteria on object characteristics in ATD leading to an increase in false alarms. Significant sources of errors in the correction step include scatter, beam hardening and the dynamic range of the DAS. These problems occur mainly when large, cluttered bags are scanned.

Because of the finite resolution of the scanner, physical objects may be fused together in the segmentation step of ATD. The characteristics of these fused objects may not be representative of the constitute objects. Artifacts caused by imperfect correction may cause the segmentation step to split objects into multiple smaller objects. The smaller objects may have masses under the mass limit. In order to detect these smaller objects, the mass threshold may have to be lowered, leading to the admission of additional nonthreats. 


\section{Involvement of Third Parties}

\subsection{Strategy for third parties}

The US Department of Homeland Security (DHS) Science \& Technology Directorate (S\&T), in coordination with the Transportation Security Administration (TSA), has identified requirements for future explosives scanners that include a larger number of threat categories, higher probability of detection per category, lower false alarm rates and lower operating costs. One tactic that DHS is pursuing to achieve these requirements is to create an environment in which the capabilities of the established scanner vendors could be enhanced or augmented by third-party algorithm development. A third-party developer in this context refers to academics, small companies and organizations other than the established scanner vendors. DHS is particularly interested in adopting the model that has been used very successfully by the medical imaging industry, in which university researchers develop algorithms that are eventually deployed in commercial medical imaging equipment. ${ }^{5}$ This model has improved the ability of radiologists to identify, locate and treat potential cancerous abnormalities.

\subsection{Opportunities for third parties}

In this section, we list potential areas where third parties could develop advanced algorithms to improve the performance of EDS equipment.

1. Reconstruction

a. Correction

b. Reconstruction including iterative/statistical reconstruction

c. Image post processing

2. Automated threat detection

a. Segmentation

b. Feature measurement

c. Classification

d. Directly from raw or corrected projection data (i.e., not using the reconstructed images)

3. Operator assist for on-screen resolution

\subsection{Data available for third parties}

The following types of data are available for third parties.

1. Raw projection data

2. Corrected projection data

3. Image data

5 When we speak of an algorithm, we are talking about the mathematical steps. The actual implementation is usually in a general purpose computer. 
In order to use the above types of data, the following supplemental data and information is available.

1. Calibration files and description of required correction steps

2. Data formats

3. Scanner geometry and other parameters required to reconstruct data

The data are available from the following types of scanners.

1. Security CT scanners (both deployed and non-deployed)

2. Medical CT scanners

3. NDE CT scanners

The scans are of the following objects.

1. Common items found in luggage

2. Geometrical shapes to allow testing of advanced algorithms

3. Explosive simulants

4. Explosives

Some of the above information may be SSI, classified or proprietary to vendors. It is beyond the scope of this document to describe how a third party receives permission to access any or all of the above cited information.

\section{Additional Information}

One tactic that DHS is using to stimulate academic and industrial third-party algorithm development is to sponsor workshops addressing the research opportunities that may enable the development of next-generation algorithms for homeland security applications. The first such workshop, entitled "Algorithm Development for Security Applications (ADSA) Workshop," was held at Northeastern University (NEU) on April 23-24, 2009. The workshop was led by Professor Michael B. Silevitch (NEU) as part of the DHS Center of Excellence (COE) for Awareness and Localization of Explosives-Related Threats (ALERT).

The main recommendation of the first workshop was to establish grand challenges for different aspects of threat detection and different screening modalities. The aspects of threat detection include (1) reconstruction and processing of sensor data, (2) image segmentation, (3) automated threat detection and (4) improved operator performance. The screening modalities include $\mathrm{x}$-ray computed tomography (CT) for checked and carry-on baggage, advanced imaging technology, cargo inspection, and stand-off detection.

It was further recommended at the first ADSA workshop that the first grand challenge should develop advanced segmentation algorithms from volumetric (CT) data for the purpose of enhancing ATD algorithms for Explosives Detection Systems and for CTbased checked baggage scanners for the check-point. 
A second ADSA workshop was held at NEU on October 7-8, 2009, under the direction of Professor Silevitch, Harry Martz (LLNL) and Carl Crawford (DHS S\&T). The purpose of the second workshop was to discuss the efforts necessary to continue investigation and development of third-party algorithms in particular how to implement a grand challenge for segmenting objects from volumetric CT data. In essence, the purpose of the second workshop was to review the plan for the CT segmentation grand challenge. The objectives the workshop were delineated by the following statement from Doug Bauer (DHS S\&T):

"Our overarching goal is to protect the American people better in travel environments against an evolving, dynamic range of threats. We need the best hardware and best algorithm development. We think that the medical field can help provide a framework for us and we brought you together for a multidisciplinary approach. How do we preserve openness to innovation? How do we meet the near-term requirements of DHS without forsaking academic research?

A third ADSA workshop was held at NEU on April 27-28, 2010, under the direction of Professor Silevitch, Carey Rappaport (NEU) and Carl Crawford (DHS S\&T). The purpose of the third workshop was to discuss how third parties could participate in the development of algorithms for advanced imaging technology, which is also known as whole body imaging.

A fourth ADSA workshop was held at NEU on October 5-6, 2010, under the direction of Professor Silevitch, Harry Martz (Lawrence Livermore National Laboratory) and Carl Crawford (DHS S\&T). The purpose of the fourth workshop was to discuss how third parties could participate in the development of reconstruction algorithms for explosive detection equipment based on CT scanning.

The reports from these above-mentioned workshops are available online as listed below [ADSA01] [ADSA02] [ADSA03] [ADSA04].

A summary of the CT-based EDS has been written by Smith and Connelly [SmithConnelly].

\section{Citations}

[ADSA01] Final Report, Algorithm Development for Security Applications Workshop, Northeastern University, April 23-24, 2009. Report available at $\mathrm{ftp}: / / \mathrm{ftp} . c e n s s i s . n e u . e d u / A D S A 01 /$ ADSA01_final_report.pdf

[ADSA02] Final Report, Algorithm Development for Security Applications Workshop, Northeastern University, October 7-8, 2009. Report available at ftp://ftp.censsis.neu.edu/ADSA02/ASDA02/final_report.pdf 
[ADSA03] Final Report, Algorithm Development for Security Applications Workshop, Northeastern University, April 27-28, 2010. Report available at ftp://ftp.censsis.neu.edu/ADSA03/ADSA03/final.pdf

[ADSA04] Final Report, Algorithm Development for Security Applications Workshop, Northeastern University, October 5-6, 2010. Report will be available shortly at ftp://ftp.censsis.neu.edu/ADSA04.

[Management-Plan] Management Plan for Explosives Detection System Certification Testing, DOT/FAA/AR-01/19.

[Kak-Slaney] Kak, A. C., and Slaney, M., Principles of Computerized Tomographic Imaging, IEEE Press, 1988.

[Smith-Connelly] R.C. Smith and J.M. Connelly, "CT Technologies," in M. Marshall and J.C. Oxley (Editors), Aspects of Explosives Detection, Elsevier B.V., 2009.

[Ying] Ying, Z., Naidu, R., and Crawford, C., "Dual Energy Computed Tomography for Explosive Detection," Journal of X-ray Science and Technology, vol. 14, 2006, pp. 235-256.

\section{Acronyms/Definitions}

\begin{tabular}{|l|l|}
\hline 2D & Two-dimensional \\
\hline 3D & Three-dimensional \\
\hline AC & Alternating current \\
\hline ADSA & Algorithm Development for Security Applications \\
\hline ADSA01 & First ADSA workshop held in April 2009 on the check-point application \\
\hline ADSA02 & $\begin{array}{l}\text { Second ADSA workshop held in October 2009 on the grand challenge for } \\
\text { CT segmentation }\end{array}$ \\
\hline ADSA03 & $\begin{array}{l}\text { Third ADSA workshop held in April 2010 on whole body imaging } \\
\text { (advanced imaging technology) }\end{array}$ \\
\hline ADSA04 & $\begin{array}{l}\text { Fourth ADSA workshop held in October 2010 on the advanced } \\
\text { reconstruction algorithms for CT-based EDS }\end{array}$ \\
\hline AIT & $\begin{array}{l}\text { Advanced imaging technology. Technology for find objects of interest on } \\
\text { passengers. WBI is a deprecated synonym. }\end{array}$ \\
\hline ALERT & $\begin{array}{l}\text { Awareness and Localization of Explosives-Related Threats, } \\
\text { A Department of Homeland Security Center of Excellence at NEU }\end{array}$ \\
\hline BVS & Baggage viewing station \\
\hline CC & Control computer \\
\hline Clearing & $\begin{array}{l}\text { The process of ATD saying that a threat is not present in a bag or that the } \\
\text { decision of ATD is overridden by secondary inspection. }\end{array}$ \\
\hline COE & Center of excellence, a DHS designation \\
\hline CRT & Certification readiness testing \\
\hline CT & Computerized tomography \\
\hline
\end{tabular}




\begin{tabular}{|l|l|}
\hline DAS & Data acquisition system \\
\hline DC & Direct current \\
\hline DOT & Department of Transportation \\
\hline DR & Digital radiology line scanner \\
\hline EDS & $\begin{array}{l}\text { Explosive detection system. The EDS is composed of a CT scanner, ATD, } \\
\text { a workstation, and a control computer. }\end{array}$ \\
\hline ETD & Explosive trace detection \\
\hline FAA & Federal Aviation Administration \\
\hline FAT & Factory acceptance test \\
\hline FBP & Filtered back-projection \\
\hline FDRS & Field data reporting system \\
\hline HVPS & High voltage power supply \\
\hline ID & Identification or identifier \\
\hline IED & Improvised explosive device \\
\hline LLNL & Lawrence Livermore National Laboratory \\
\hline Mis-track & A bag that cannot be tracked by the BHS \\
\hline NEU & Northeastern University \\
\hline ODT & Ordinance disposal team \\
\hline OSARP & On-screen alarm resolution protocol \\
\hline OSR & On-screen resolution \\
\hline PD & Probability of detection \\
\hline PFA & Probability of false alarm \\
\hline PVS & Primary viewing station \\
\hline SAT & Site acceptance test \\
\hline Shield & $\begin{array}{l}\text { The condition when the EDS cannot view a portion of a bag because the x- } \\
\text { ray beam is extinguished of the presence of clutter. }\end{array}$ \\
\hline SOP & Standard operating procedure \\
\hline SSI & Sensitive security information \\
\hline SVS & Secondary viewing station \\
\hline Threat & A portion of a bag that is a potential threat as determined by the ATD \\
\hline TSL & Transportation Security Laboratory, Atlantic City, NJ \\
\hline TSO & $\begin{array}{l}\text { Transportation security officer: operator of the BVS and worked in the } \\
\text { BIR }\end{array}$ \\
\hline WBI & Whole body imaging; a deprecated term for AIT \\
\hline XRD & X-ray diffraction \\
\hline & \\
\hline
\end{tabular}




\section{Figures}

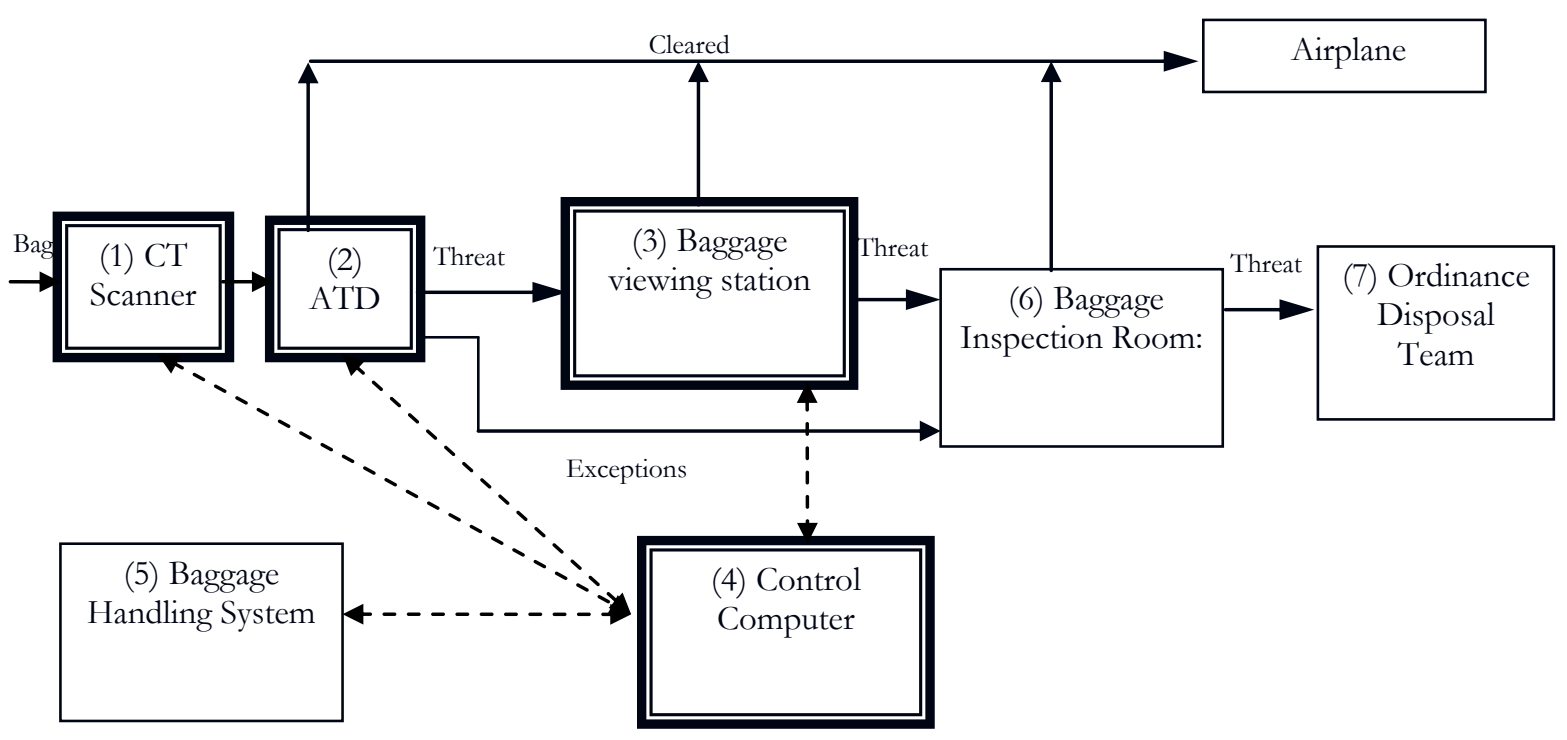

Figure 1. Schematic diagram of an in-line EDS. Wide-lined boxes are components of EDS. Narrow-lined boxes are sub-systems used in conjunction with the EDS. Solid connecting lines show flow of bags and/or images of the bags. Dashed connecting lines show the flow of control and information.

Automated Threat Detection

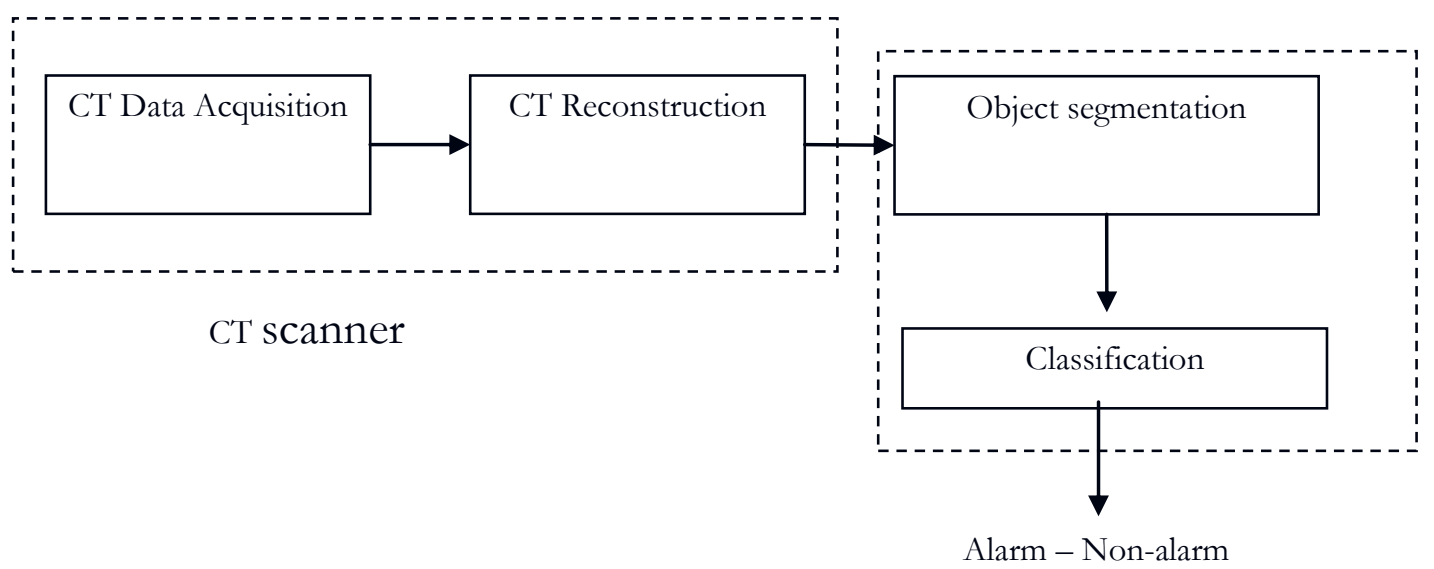

Figure 2. Schematic diagram of a CT-based EDS including ATD. 


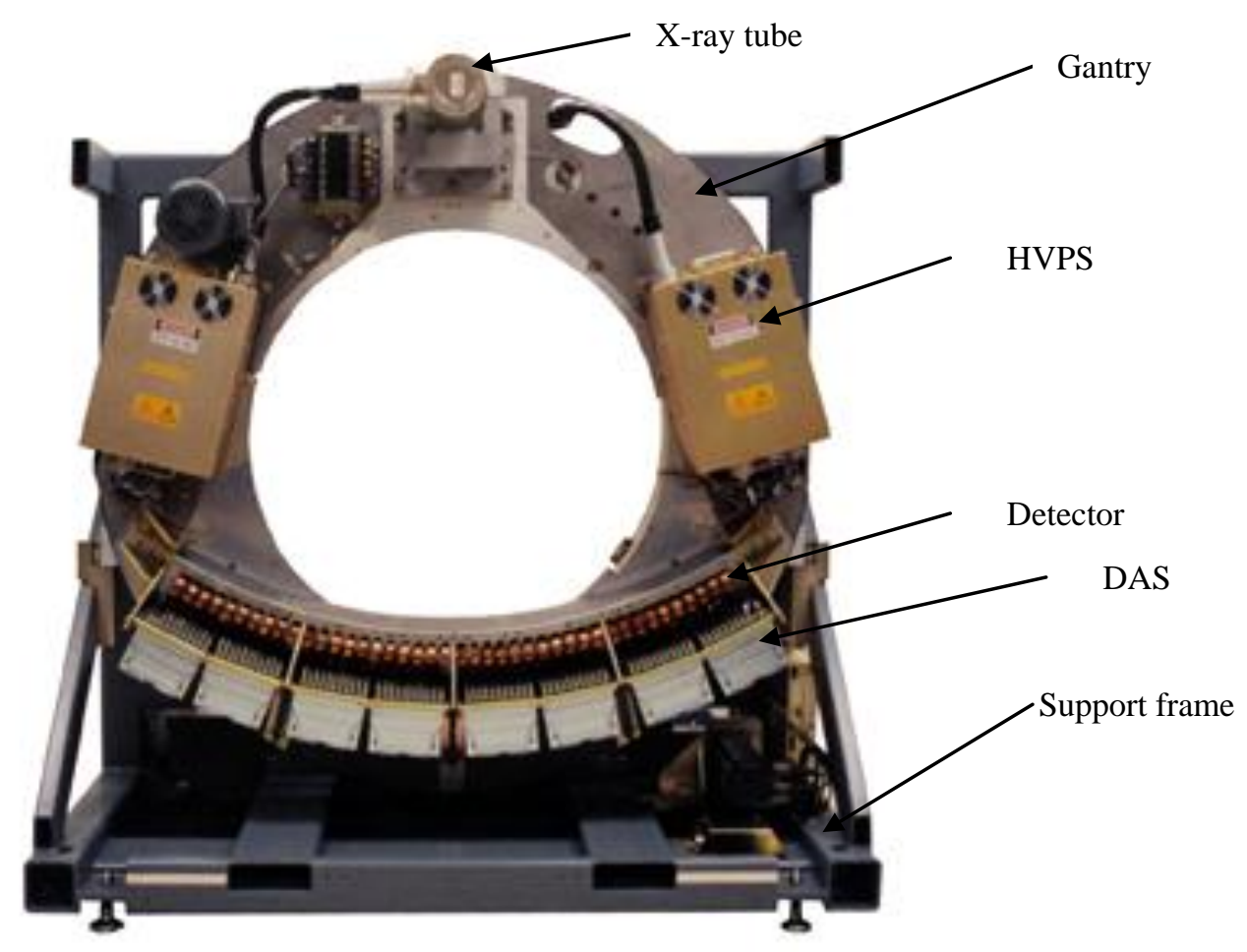

Figure 3. Picture of the inside of a L-3 eXaminer 6000. The annotated portions show the components of the X-ray beam line. [Permission granted from Analogic to reprint this figure.]

DAS

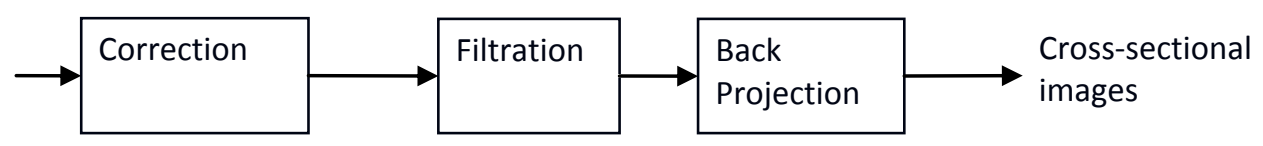

Figure 4. A schematic diagram of the CT reconstruction process. 


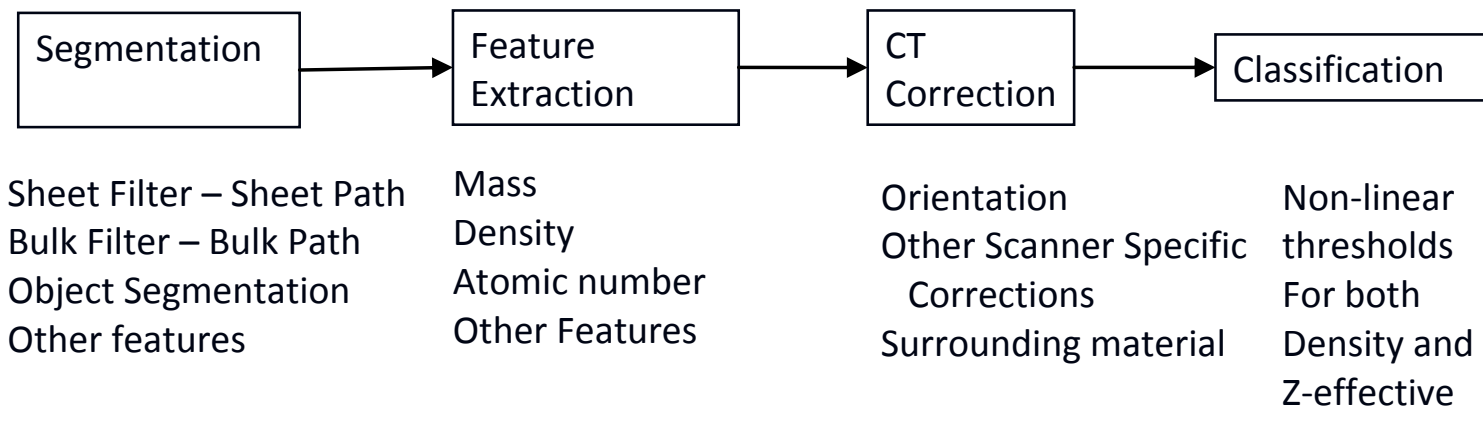

Figure 5. Simplified schematic diagram of one possible version of ATD.

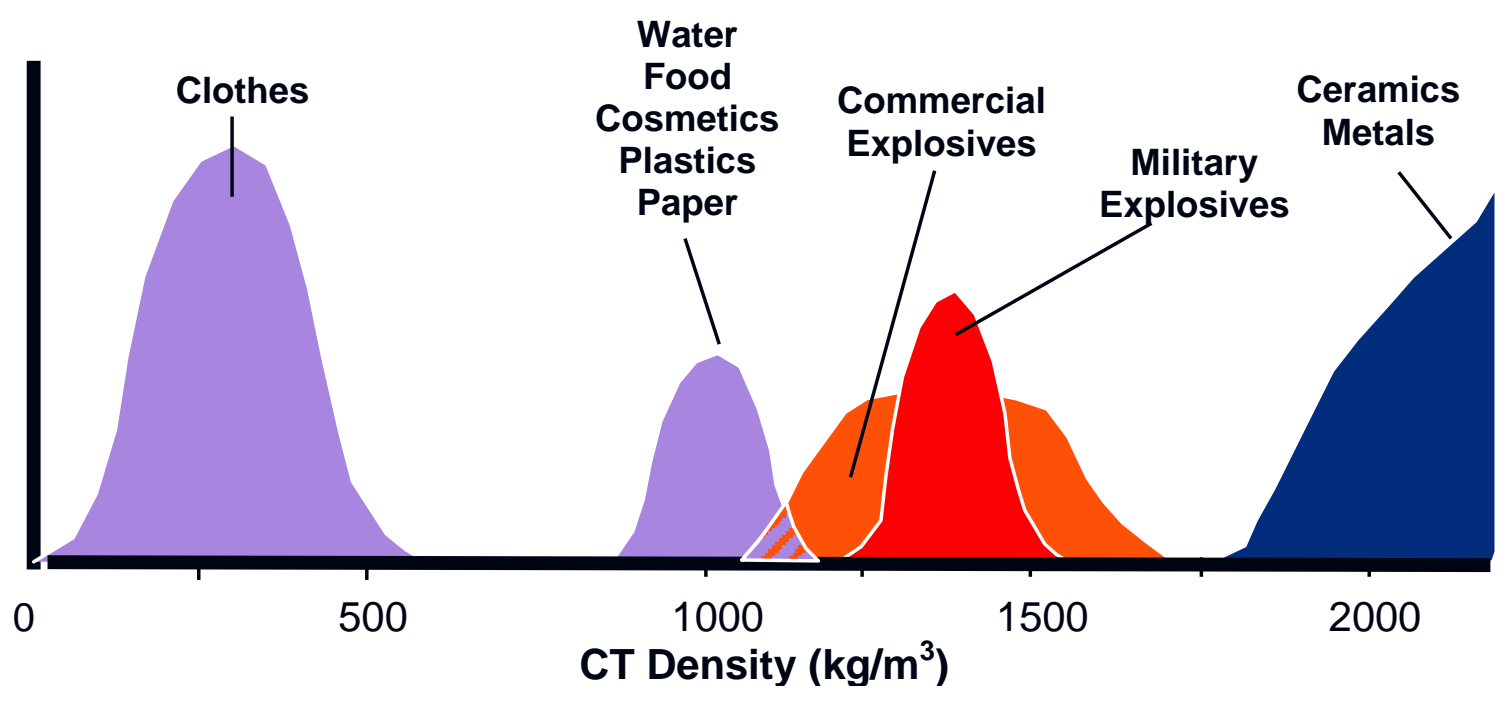

Figure 6. Overlap of threats and non-threats using in CT density space. 


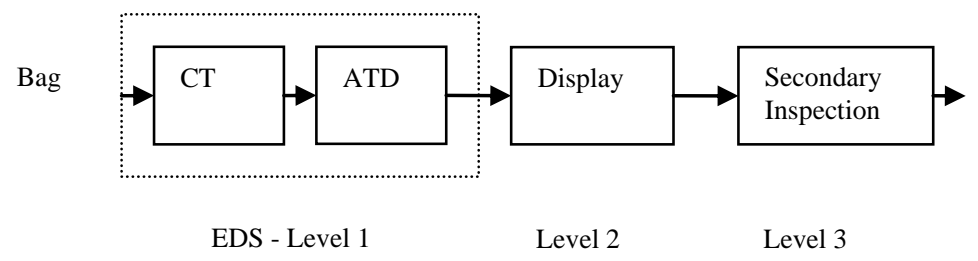

Figure 7: Schematic of an EDS and additional levels of screening used to resolve alarms from the ATD. 


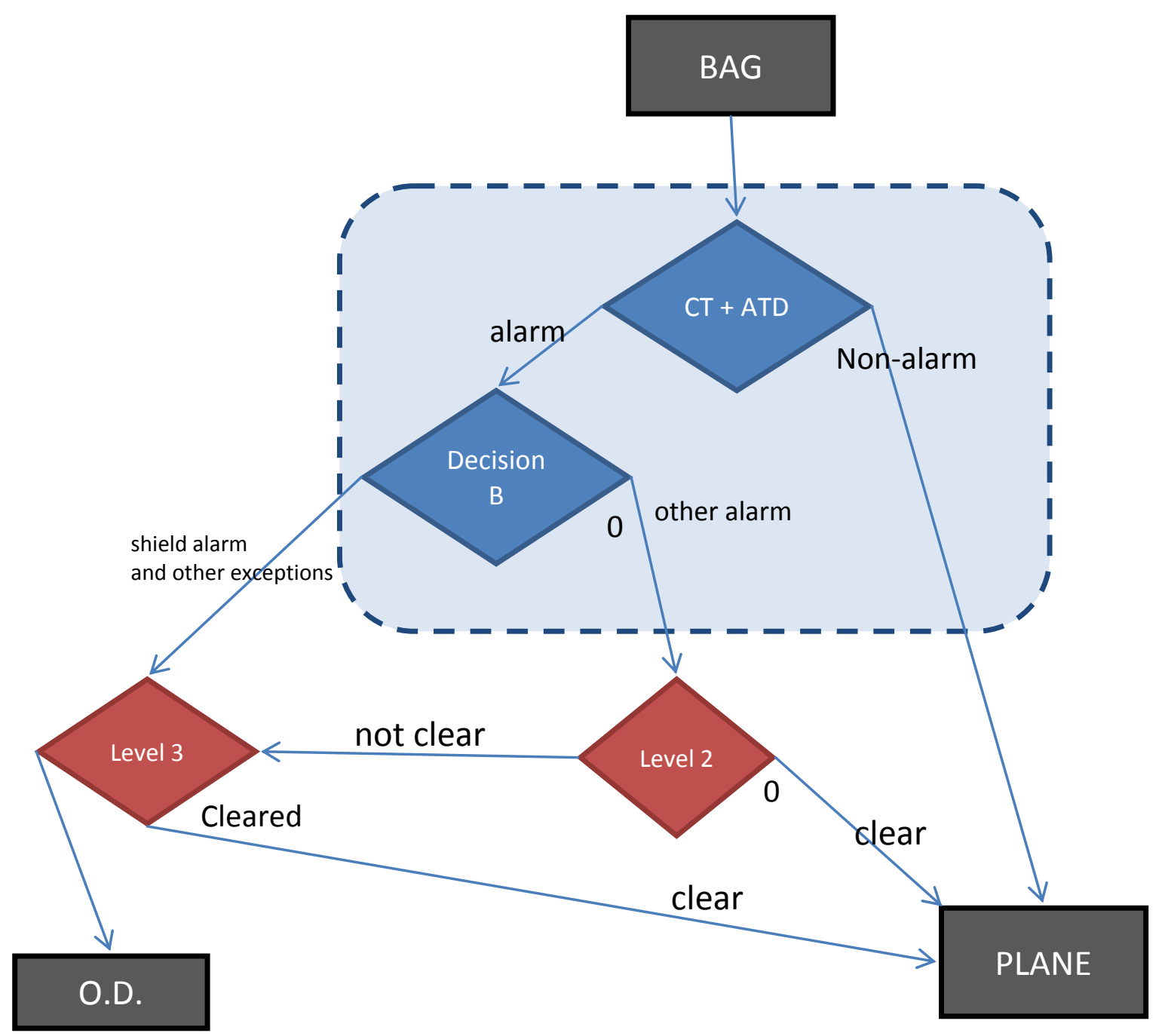

Figure 8: Schematic diagram of the decision process. 


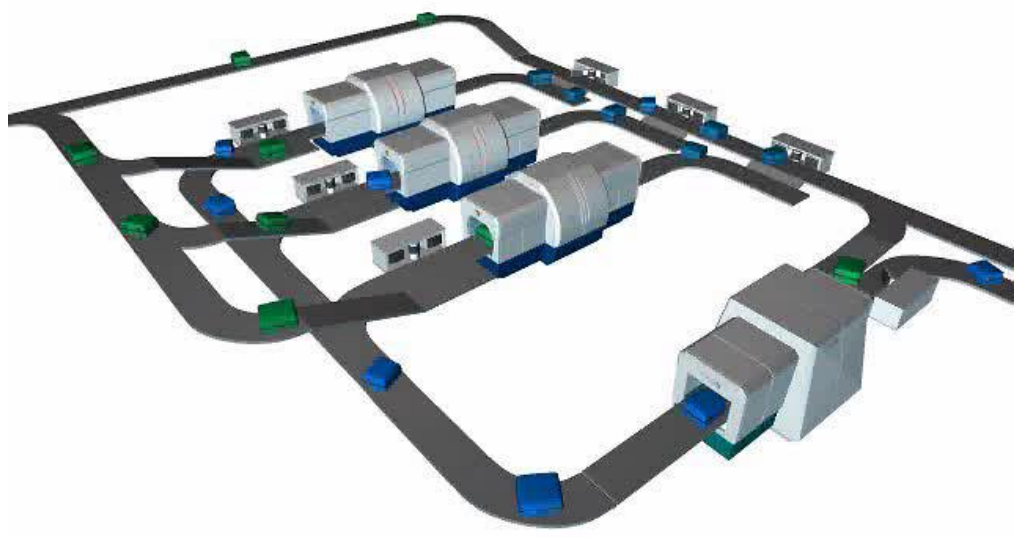

Figure 9. A schematic diagram of four EDSs deployed in an inline configuration. 